\title{
BRIDGE OVER TROUBLED WATER? \\ es Stanford MEDICINE UTILITY OF PARAVERTEBRAL BLOCKADE IN MYOCARDIAL BRIDGE UNROOFING
}

\author{
J Boublik*, S. Mistry ${ }^{1}$, B. Tsui ${ }^{1}$, J. Boyd ${ }^{2}$, J. Brodt ${ }^{1}$, JL Horn ${ }^{1}$
}

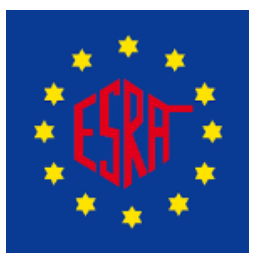

*Department of Anesthesiology, Perioperative, and Pain Medicine, ${ }^{* *}$ Department of Cardiothoracic Surgery Stanford University, Palo Alto, California, United States

\section{Background:}

Myocardial bridges are bands of myocardium overlapping coronary arteries, symptomatic bands require surgery due to their potential of morbidity ${ }^{1}$. Incidence of chronic pain after thoracotomy $y^{2}$ and sternotomy ${ }^{3}$ are up to 33 and $56 \%$ respectively Paravertebral blocks (PVB) provide effective analgesia in cardiothoracic surgery ${ }^{4,5}$.

Retrospective analysis to investigate the utility with respect to healthcare resource utilization.

\section{Methods:}

Case series of 98 patients undergoing myocardial bridge unroofing- 72 patients (73.5\%) sternotomy and $26(26.5 \%)$ thoracotomy. For data analysis, sternotomy and thoracotomy groups separated. Sternotomy group: 38 no block and 29 received bilateral PVB's, 5 were excluded.

Thoracotomy group: 19 patients unilateral PVB, 6 no blocks, one patient excluded after receiving liposomal Bupivacaine.

\section{Results}

Sternotomy patients receiving PVB's had a significantly shorter intensive care unit (ICU) stay, total length of stay was unaffected

Thoracotomy patients receiving PVBs had shorter ICU admissions (and hospitalizations than patients not receiving blocks

\section{Conclusion}

Paravertebral blocks are of potential utility in cardiothoracic surgery for myocardial bridge unroofing. Depending on the surgical approach, they facilitate either shorter ICU admissions or shorter ICU and hospital stays and decrease health care resource utilization.

\section{References:}

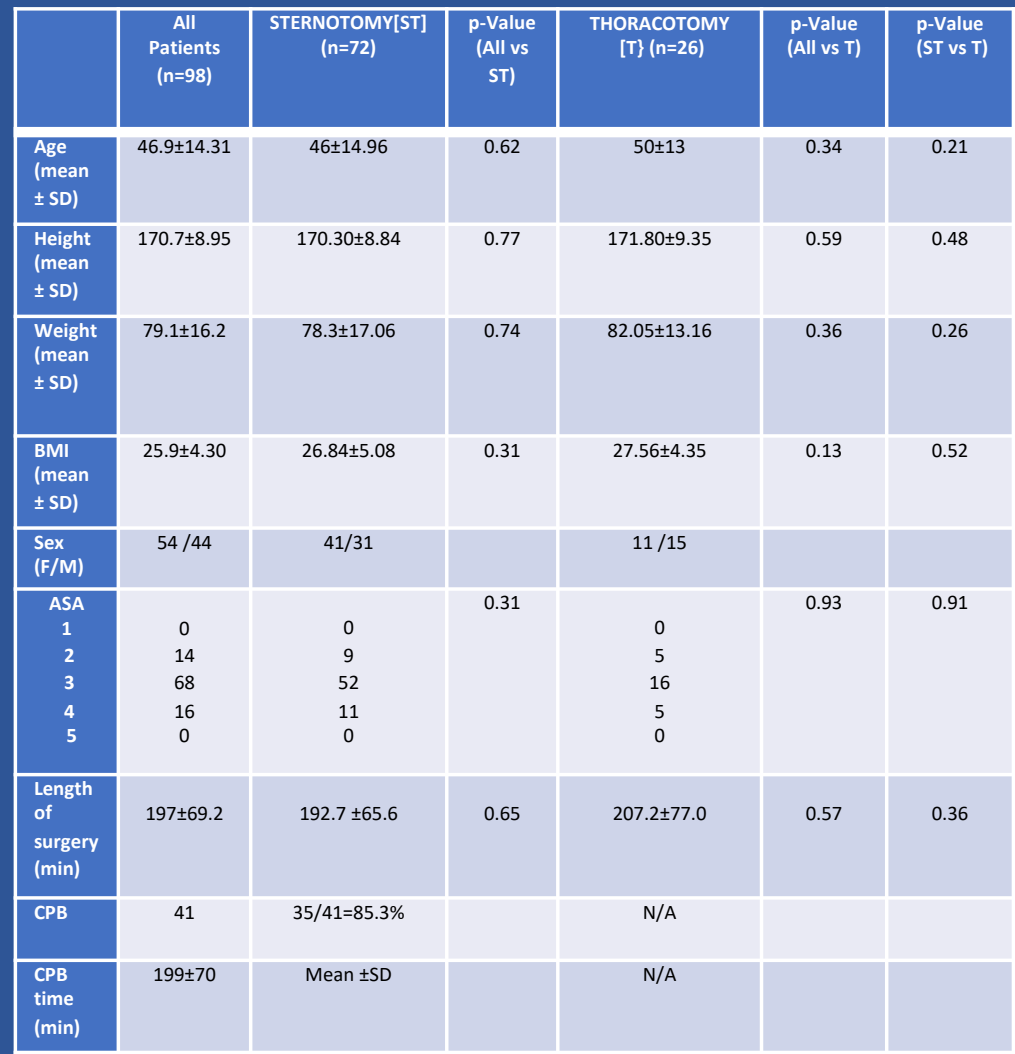

\section{Sternotomy patients}

\begin{tabular}{|c|c|c|c|}
\hline & $\begin{array}{c}\text { Group } \\
\text { CPB- No Block } \\
(\mathrm{N}=38)\end{array}$ & $\begin{array}{c}\text { Group } \\
\text { CPB-PVB } \\
(\mathrm{N}=29)\end{array}$ & p-values \\
\hline LOS ICU & $1.60 \pm 0.82$ & $1.37 \pm 0.56$ & $\mathbf{0 . 0 2}$ \\
\hline LOS hospital & $4.41 \pm 1.14$ & $4.23 \pm 1.04$ & 0.07 \\
\hline
\end{tabular}

\section{Thoracotomy patients}

\begin{tabular}{|c|c|c|c|}
\hline & $\begin{array}{c}\text { Group No } \\
\text { Block (N=6) }\end{array}$ & $\begin{array}{c}\text { Group PVB } \\
(\mathrm{n}=19)\end{array}$ & p-values \\
\hline LOS ICU & $2.2 \pm 0.74$ & $1.21 \pm 0.71$ & $\mathbf{0 . 0 2}$ \\
\hline LOS hospital & $4.16 \pm 0.75$ & $3.73 \pm 1.04$ & $\mathbf{0 . 0 0 2}$ \\
\hline
\end{tabular}

1. Maeda K, Schnittger I, Murphy DJ, Tremmel JA, Boyd JH, Peng L, Okada K, Pargaonkar VS, Hanley FL, Mitchell RS, Rogers IS. Surgical unroofing of hemodynamically significant myocardial bridges in a pediatric population. J Thorac Cardiovasc Surg. 2018 Oct; 156(4):1618-1626

2. Bayman EO, Parekh KR, Keech J, Selte A, Brennan TJ. A Prospective Study of Chronic Pain after Thoracic Surgery. Anesthesiology. 2017;126(5):938-951

3. Kleiman AM, Sanders DT, Nemergut EC, Huffmyer JL. Chronic Poststernotomy Pain:Incidence, Risk Factors, Treatment, Prevention, and the Anesthesiologist's Role. Reg Anesth Pain Med. 2017 Nov/Dec;42(6):698-708

4. EI Shora HA, El Beleehy AA, Abdelwahab AA, Ali GA, Omran TE, Hassan EA, Arafat AA. Bilateral Paravertebral Block versus Thoracic Epidural Analgesia for PainControl Post-Cardiac Surgery: A Randomized Controlled Trial. Thorac Cardiovasc Surg. 2018 Aug 16. doi: 10.1055/s-0038-1668496. [Epub ahead of print]

5. Komatsu T, Sowa T, Takahashi K, Fujinaga T. Paravertebral block as a promising analgesic modality for managing post-thoracotomy pain. Ann Thorac Cardiovasc Surg. 2014;20(2):113-6. 